\title{
INTRODUCTION AND EVALUATION OF A DAMPING DETERMINATION METHOD BASED ON THE SHORT-TERM FOURIER TRANSFORM AND RESAMPLING (STFR)
}

\author{
Olaf Peter Hentschel, Lars Panning-von Scheidt, Jörg Wallaschek \\ Leibniz Universität Hannover, Institute of Dynamics and Vibration Research, Hannover, Germany \\ e-mail: hentschel@ids.uni-hannover.de; panning@ids.uni-hannover.de; wallaschek@ids.uni-hannover.de \\ MARKUS DENK \\ ALSTOM Power, Steam Turbines and Generators, Baden, Switzerland \\ e-mail: markus.denk@power.alstom.com
}

\begin{abstract}
In the present paper, a frequency domain method for damping determination is presented. The described method is especially developed for low damped systems with well separated eigenfrequencies. Using the Short-Term Fourier transform and Resampling (STFR) of the signal, decay curves of several mode shapes can be identified and amplitude-dependent damping values can be calculated. Additionally, two common methods for damping determination are explained briefly. Finally, the quality of the introduced method is evaluated comparing the variances of the identified damping values by means of different methods. In this context, the damping for beams clamped in a suspended way is analyzed. Stainless steel is used as the specimen material.
\end{abstract}

Keywords: damping determination, parameter identification, resampling, nonlinear dynamic analysis

\section{Introduction}

In fields of mechanical engineering, High Cycle Fatigue (HCF) risk assessment is of great importance. To reduce this risk, the reduction and prediction of vibration amplitudes is a primary objective. Using nonlinear calculation tools (Petrov and Ewins, 2006; Siewert et al., 2008) to predict vibration amplitudes, the specification of contact properties as well as structural damping (among other mechanisms of damping, Rao and Saldanha, 2003) are of great importance. To specify, for example, structural damping values, a suitable experimental setup and an appropriate methodology for damping identification are elementary. In this context, Plunkett (1959) gives an overview of possible methods (time and frequency domain) for damping determination. Furthermore, Bert (1973) offers an overview of methods for modeling, experimental determination and parameter identification of material damping. An extended Half-Power method is described by Jinting et al. (2013), whereby the parameter identification is performed in the frequency domain. In addition, Yang et al. (2003), Meissner (2012) and Feldman (1994) describe a method applying the Hilbert transform to calculate decay curves. By Yang et al. (2011), Lardies and Gouttebroze (2002), Lamargue et al. (2000), Hans et al. (2000), Le and Argoul (2004) and Slavi et al. (2003), the wavelet transform is applied for parameter identification.

The methodology which is presented in this paper is limited to structures excited by an impact. Additionally, the eigenfrequencies, respectively the resonant frequencies have to be well separated. The described method includes the Short-Term Fourier transform and Resampling (STFR) of the analyzed signal. This procedure makes it possible to get adequate information concerning the vibration amplitude and the current eigenfrequency which varies as a function 
of amplitude for nonlinear mechanical systems. On this basis, it is possible to identify the decay curve of one selected mode shape of interest. Using a decay fit (Rice et al., 2007; Siewert et al., 2008 ), it is possible to identify the damping value of one selected mode shape. Subsequently, two other common methods for parameter identification are presented. Furthermore, an experimental setup is introduced briefly, which provides the experimental data for damping determination. Finally, the presented methods are used to evaluate the developed STFR method in the last part of this paper.

\section{STFR method}

The presented method is used to identify damping values of mechanical systems at decaying free vibrations. This method is restricted to impulse excited structures. In this context, only the output signal is needed for damping identification. To achieve an adequate identification concerning the eigenfrequency and amplitude, the signal is additionally resampled. From this, tracking of the eigenfrequency depending on the amplitude is also possible.

\subsection{Short-Term Fourier transform and Resampling}

The basis for the used method is the measured output signal. In this case, the specimen is excited by an impact, whereby decay curves are recorded. The signal $x$ can be specified by the following data sequence with $N_{s}$ values.

$$
x[n]= \begin{cases}x\left(t=n T_{0}\right) & \text { for } 0 \leqslant n \leqslant N_{S}-1 \\ 0 & \text { otherwise }\end{cases}
$$

wherein $T_{0}, t$ and $n$ denote the sample rate, the time and the value number. From Fig. 1, the superimposed character of the signal (response of several mode shapes due to impact excitation) can be clearly seen.

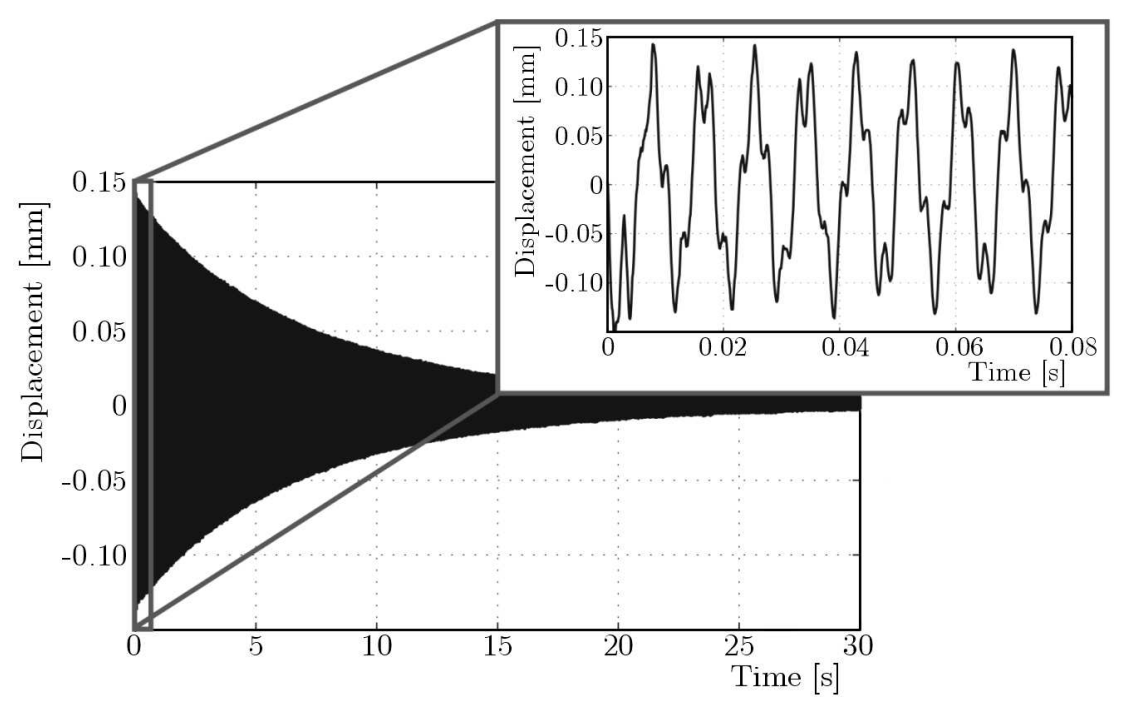

Fig. 1. Measured free vibration

In the next step, the signal has to be extracted with respect to one mode shape of interest. Therefore, the signal is analyzed stepwise. The signal is equidistantly divided into

$$
U=\frac{N_{S}}{N_{W}}-1
$$


parts. For these parts of the decaying signal, the Discrete Fourier Transform (DFT) is performed. $N_{W}$ describes the number of measurement points of the analyzed signal part. The following $u$ signal portions with $0 \leqslant u \leqslant U$ are resulting in

$$
x_{u}[w]= \begin{cases}x\left(t=\left(u N_{w}+w\right) T_{0}\right) & \text { for } 0 \leqslant w \leqslant N_{w}-1 \\ 0 & \text { otherwise }\end{cases}
$$

wherein $w$ denotes the value number of the signal portion. Next, the data portion $x_{u}[w]$ has to be transformed into frequency domain

$$
X_{u}[k]=\sum_{w=0}^{N_{w}-1} x\left(\left(u N_{w}+w\right) T_{0}\right) \mathrm{e}^{-j 2 \pi \frac{k w}{N_{w}}}
$$

wherein $k$ is the spectral line number. Using this formulation, the frequency resolution reads

$$
\Delta \omega_{f}=\frac{2 \pi}{T_{0} N_{w}}
$$

This leads to the problem that the amplitude at the eigenfrequency is underestimated, in particular for weakly damped structures. Performing a resampling of the analyzed data portion solves this problem. The goal in this context is the identification of the required sample rate in a way that the frequency resolution $\Delta \omega_{f, n}$ is equal to an integer numbered multiple $g$ of the eigenfrequency of interest $\omega_{d \text {,int }}$

$$
\omega_{d, \text { int }}=g \Delta \omega_{f, n}
$$

To achieve the required sample rate corresponding to the criteria in equation (2.6), a real numbered alternation $d$ of the sample rate $T_{0}$ is necessary

$$
\Delta \omega_{f, n}=\frac{2 \pi}{T_{0, n} N_{w}}=\frac{2 \pi}{d T_{0} N_{w}}
$$

From this, an integral numbered decimation using the factor $a$ followed by an integral numbered interpolation using the factor $b$ is performed. Hereby, a resampling using the real factor $d=a / b$ is realized. To avoid aliasing, the Nyquist-Shannon sampling theorem has to be satisfied. In this respect, a lowpass filter regarding the varied sample rate is used. This lowpass filter avoids the aliasing effect at decimation and interpolates the signal at interpolation. The resampling process (Oppenheim and Schafer, 2010) is shown in Fig. 2.

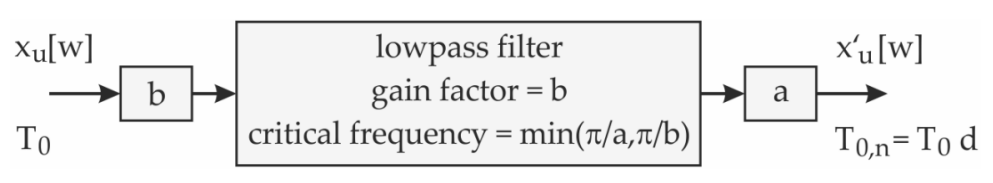

Fig. 2. Resampling

\subsection{Methodology}

The presented methodology is based on the assumption of well separated eigenfrequencies. Thus, the knowledge concerning the position of one eigenfrequency, respectively the frequency of the mode shape of interest, is important. In general, the exact eigenfrequency, which is the criterion for the described resampling methodology, is not known. For this purpose, an identification of the exact eigenfrequency using an optimization method (such as the Nelder-Mead algorithm, 


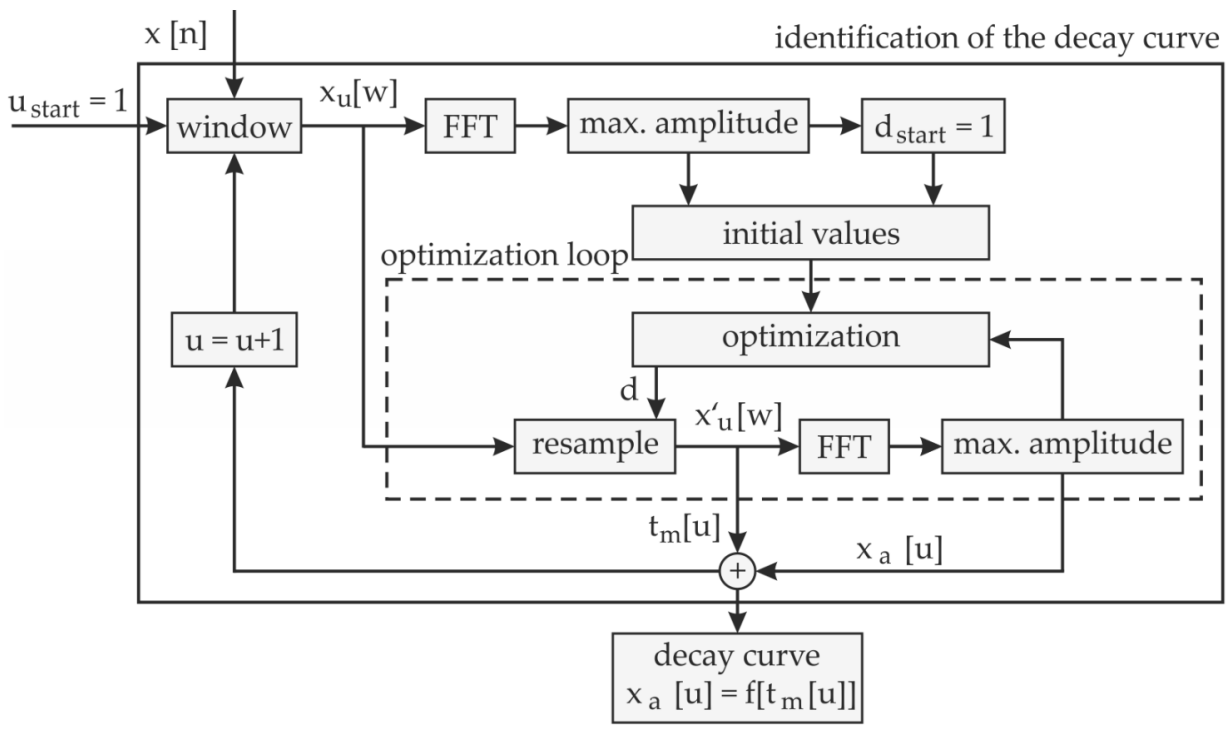

Fig. 3. Methodology

Nelder and Mead, 1965) is necessary. The resampling factor $d$ is optimized to detect the maximum amplitude within the analyzed signal portion $u$. In Fig. 3, the described methodology to calculate the decay curve is shown for one mode shape of interest.

After calculating the eigenfrequency using the described methodology, the modal amplitude for each part of the decay curve can be isolated. Calculating the average time

$$
t_{m}[u]=(u-1) N_{w} T_{0}+\frac{N_{w}}{2} d_{u} T_{0}
$$

for each part of the decay curve $u$ (Fig. 4), it is possible to reconstruct the decay curve for one mode shape of interest using the identified amplitude $x_{a}[u]$.

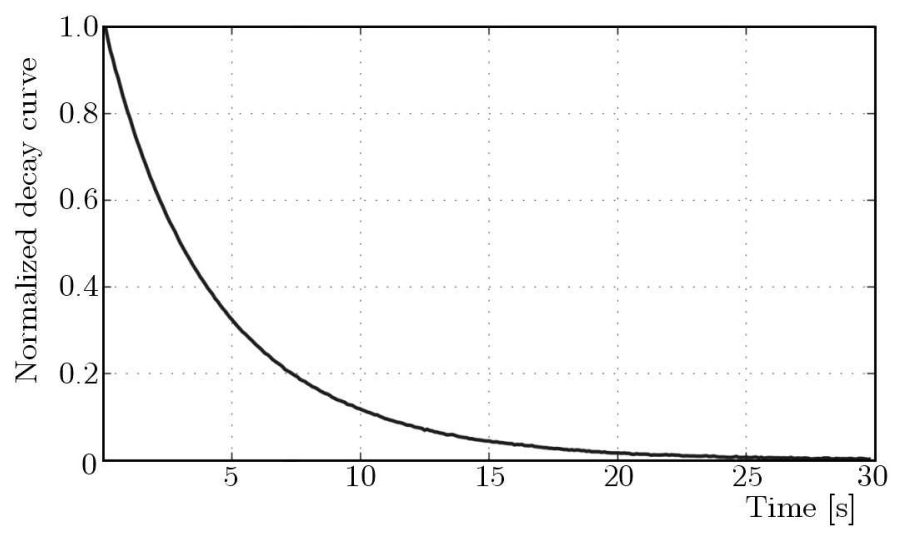

Fig. 4. Identified decay curve

To enable a quantification of a damping parameter, an analytical formulation of the decay curve is necessary. Using the least squares method, the decay coefficient $\delta$ can be determined

$$
\sum_{u=0}^{U}\left(x\left[t_{m}[u]\right]-\widehat{x}_{a} \mathrm{e}^{-\delta t_{m}[u]}\right)^{2} \rightarrow \min
$$

In equation (2.9), $\widehat{x}_{a}$ denotes the initial amplitude of the decaying signal. Performing the signal resampling, an additional advantage is present. In this context, the eigenfrequency for each time 
point of the decay curve can be calculated. Hereby, it is possible to examine the dependency of frequency and time. From the frequency versus time history, nonlinearities with respect to variation in frequency can be identified. In Fig. 5, two possible frequency-time curve shapes are presented.

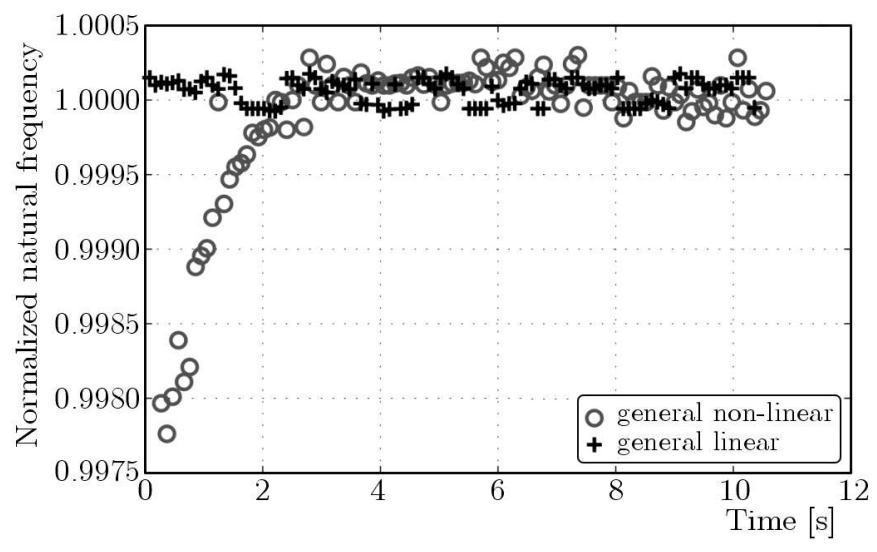

Fig. 5. Frequency versus time

\subsection{Amplitude-dependent damping coefficient}

To identify an amplitude-dependent damping, the identified decay curve has to be divided into several parts $p$. From this, the decay coefficient $\delta_{m}$ can be calculated as a function of the mean amplitude $x_{p}$ (see Fig. 6)

$$
x_{p}\left[t_{m, u}\right]=\widehat{x}_{a, p} \mathrm{e}^{-\delta\left[x_{p}\right] t_{m}[u]}
$$

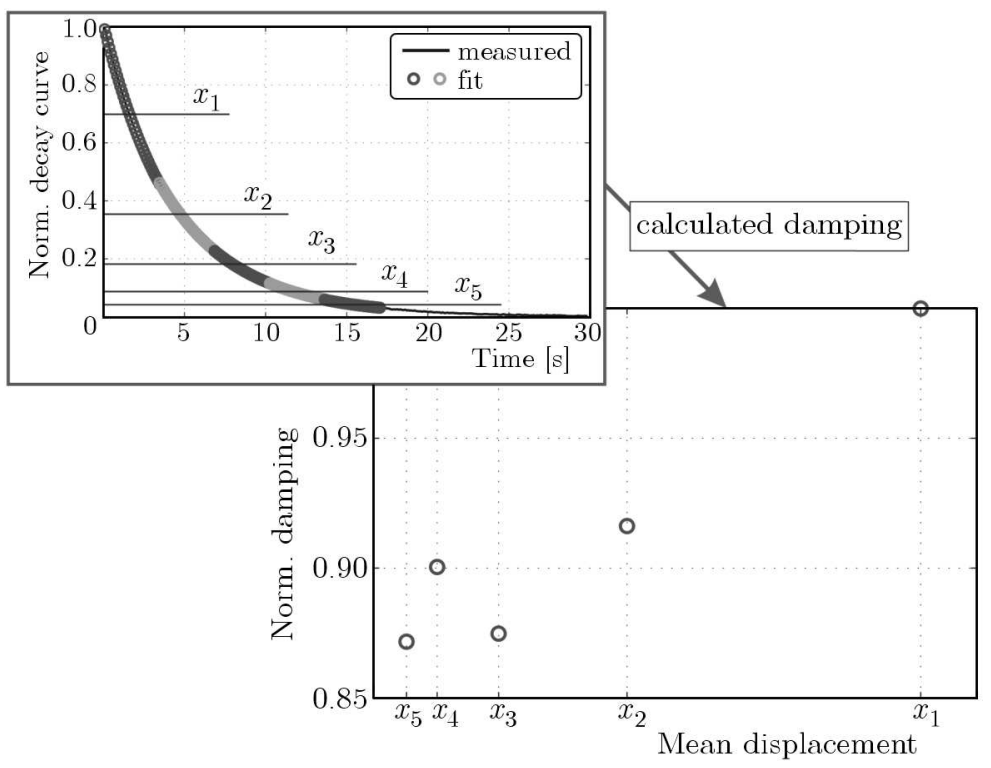

Fig. 6. Amplitude-dependent normalized mean damping coefficient

In addition to the division of the decay curve to calculate amplitude-dependent damping values, also an analytical approach is proposed. On this basis, the decay coefficient described in equation (2.9) is replaced by a time-dependent, respectively amplitude-dependent decay coefficient $\delta_{t}(t)$

$$
x\left[t_{m, u}\right]=\widehat{x}_{a} \mathrm{e}^{-\delta_{t}\left(t_{m}[u]\right) t_{m}[u]}
$$


In the next step, the time derivative of the presented decay curve is calculated. Following the calculated time derivative of the decay curve, $\dot{x}$ is divided by the decay curve itself. Hereby, an inhomogeneous first order differential equation results

$$
-\frac{1}{t} \frac{\dot{x}}{x}=\dot{\delta}_{t}+\frac{1}{t} \delta_{t}
$$

After separation of the variables and the solution of the homogeneous differential equation, a variation of the constants is performed to solve the differential equation. The analytical solution to equation (2.12) is the time-dependant decay coefficient

$$
\delta_{t}(t)=-\frac{1}{t} \int \frac{\dot{x}}{x} d t+\frac{C}{t}
$$

The constant $C$ can be calculated using a signal part $t_{s}$ of the decay curve. For this purpose, it can be assumed that the average decay coefficient through this signal part is equal to the nonamplitude-dependent decay coefficient $\delta_{m, t_{s}}$. The latter can be calculated using the formulation in equation (2.9) applying the least squares method

$$
C=\left(\delta_{m, t_{s}}\left(\max \left(t_{s}\right)-\min \left(t_{s}\right)\right)+\int_{\min \left(t_{s}\right)}^{\max \left(t_{s}\right)}\left(\frac{1}{t} \int \frac{\dot{x}}{x} d t\right) d t\right)\left(\ln \frac{\max \left(t_{s}\right)}{\min \left(t_{s}\right)}\right)^{-1}
$$

Using the prior identified eigenfrequency $\omega_{d}(t)$ as a function of time and the time-dependent decay coefficient, damping can be quantified by different physical magnitudes

$$
\Lambda(t)=2 \pi \frac{\delta(t)}{\omega_{d}(t)}=2 \pi \frac{D(t)}{\sqrt{1-D(t)}}
$$

In equation (2.15), $\Lambda(t)$ and $D(t)$ denote the logarithmic decrement and the damping ratio. Applying the amplitude formulation in equation (2.10), the time-dependent damping values can be specified in terms of amplitude.

\subsection{Example of application}

To clarify the presented methodology, a predefined amplitude-time curve is analyzed. For this reason, a time-dependent, i.e. amplitude-dependent, damping and frequency value is used

$$
x(t)=\widehat{x}_{1} \mathrm{e}^{-\delta_{1}(t) t} \cos \left(\omega_{1}(t) t\right)+\widehat{x}_{2} \mathrm{e}^{-\delta_{2}(t) t} \cos \left(\omega_{2}(t) t\right)+\widehat{x}_{3} \mathrm{e}^{-\delta_{3}(t) t} \cos \left(\omega_{3}(t) t\right)
$$

The signal consists of three frequencies, whereby the second one may be of interest. The other signal parts can be treated as additional mode shapes which are not of interest for the analysis. In this context, $\widehat{x}_{i}, \delta_{i}(t)$ and $\omega_{i}(t)$ denote the initial amplitude, the decay coefficient and the vibration frequency of each signal part. In Table 1, the magnitudes of the different values are summarized. Hereby, the units millimeters and seconds are used.

In the first step, the presented signal is transformed stepwise into frequency domain. The signal is resampled stepwise with respect to the analyzed mode shape. The considered frequency range has to consist only of the mode shape of interest. From this, an optimization of the amplitude with respect to the maximum amplitude in the analyzed signal portion is performed. In Fig. 7, one signal portion before resampling and after resampling and the resulting timefrequency-amplitude diagram are shown.

With respect to the time-frequency axis, the eigenfrequency variation can be identified. In doing so, the time dependency of the identified and the default eigenfrequency of the second signal portion are shown in Fig. 8. 
Table 1. Summarized specifications of equation (2.16)

\begin{tabular}{|c|c|c|c|}
\hline$i$ & $\widehat{x_{i}}[\mathrm{~mm}]$ & $\delta_{i}(t)\left[\frac{1}{\mathrm{~s}}\right]$ & $\omega_{i}(t)\left[\frac{\mathrm{rad}}{\mathrm{s}}\right]$ \\
\hline \hline 1 & $10 \mathrm{~mm}$ & $1.2 \frac{1}{\mathrm{~s}}-0.05 \frac{1}{\mathrm{~s}^{2}} t$ & $2 \pi \cdot 400 \frac{1}{\mathrm{~s}}+\frac{1}{\mathrm{~s}^{2}} t$ \\
\hline 2 & $8 \mathrm{~mm}$ & $0.6 \frac{1}{\mathrm{~s}}-0.045 \frac{1}{\mathrm{~s}^{2}} t$ & $2 \pi \cdot 500 \frac{1}{\mathrm{~s}}+2 \frac{1}{\mathrm{~s}^{2}} t$ \\
\hline 3 & $11 \mathrm{~mm}$ & $3 \frac{1}{\mathrm{~s}}-0.05 \frac{1}{\mathrm{~s}^{2}} t$ & $2 \pi \cdot 650 \frac{1}{\mathrm{~s}}+\frac{1}{\mathrm{~s}^{2}} t$ \\
\hline
\end{tabular}

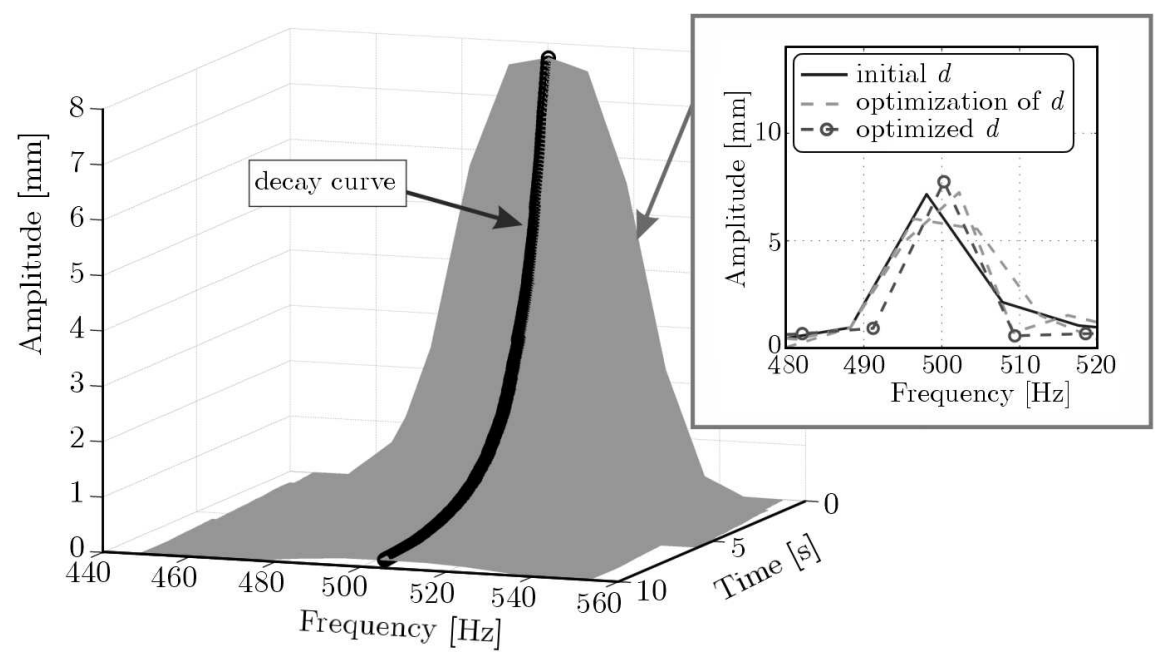

Fig. 7. Time-frequency-amplitude curve and signal resampling

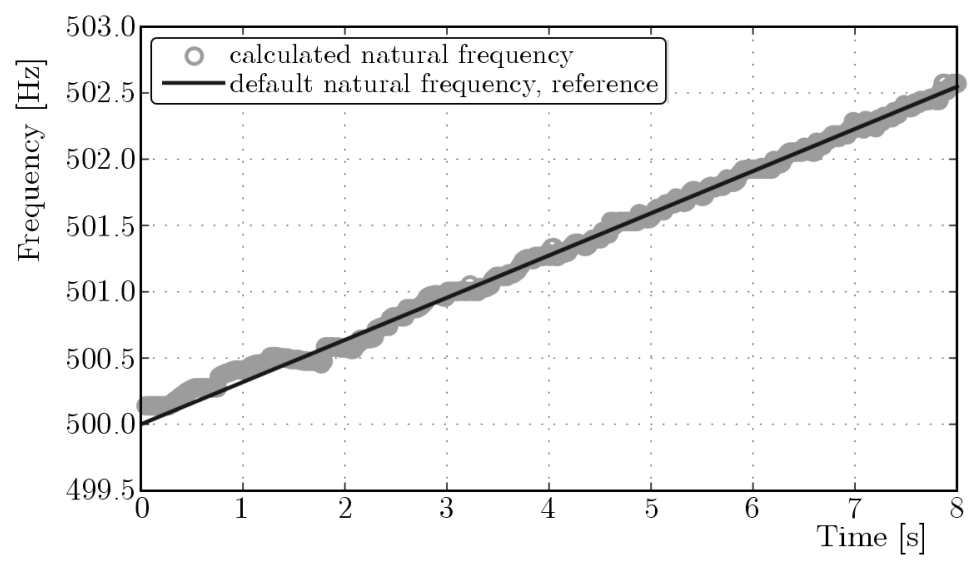

Fig. 8. Eigenfrequency versus time

In this figure, it is shown that the eigenfrequency time dependency is estimated very well. Regarding the time-amplitude axis, the decay curve of one mode shape of interest can be analyzed. Applying the developed methodology to identify amplitude-dependent damping values (presented in Section 2.3), the damping-time (respectively decaying amplitude) dependency can be analyzed (see Fig. 9).

Accordingly to the well estimated eigenfrequency behavior, the damping values can be evaluated in a suitable way. On this basis, it can be shown that the frequency and damping variation are investigable in their magnitudes and courses. 


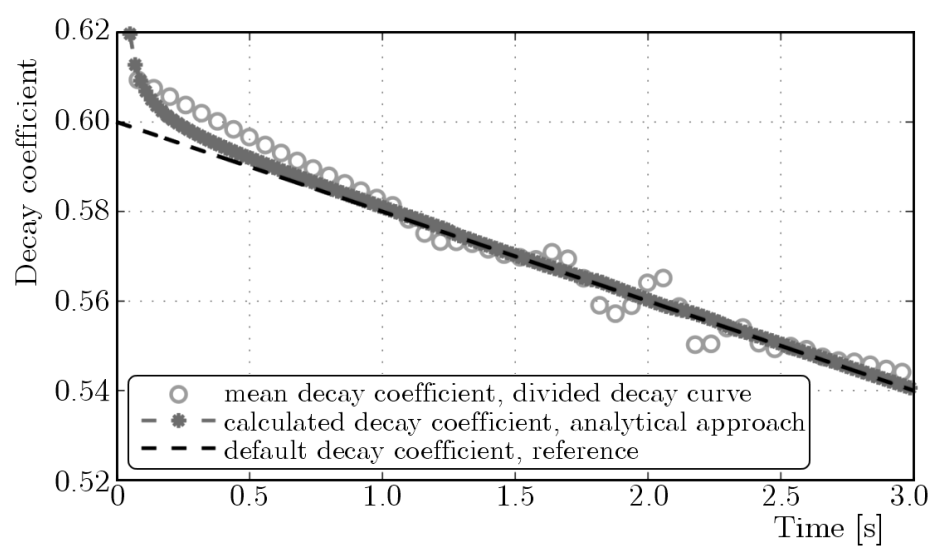

Fig. 9. Decay coefficient versus time

\section{Common methods for damping determination}

In this Section, two common methodologies for damping determination used in modal analysis are presented. For this purpose, an estimation of the transfer function is necessary. The estimated transfer function is the basis for the presented methods. In this context, the Rational Fractional Polynomials method (Richardson and Formeti, 1982) and the Least-Squares Complex Exponential method (He and Fu, 2001) are described. In the final step, the damping values determined by the developed methodology are compared to the damping values identified by the RFP and the LSCE method respectively.

\subsection{Estimation of the transfer function}

Due to noise which occurs in the measured input $y(t)$ and output $x(t)$ signal, an estimation of the Frequency Response Function (FRF) is necessary. Hereby, several FRF estimators are usable. To determine the estimators, the cross-spectrum $\left(S_{X Y}\right.$ and $\left.S_{Y X}\right)$ and the auto-spectrum $\left(S_{Y Y}\right.$ and $\left.S_{X X}\right)$ of the input and output signals are used. In Table 2, the common FRF estimation functions are presented.

Table 2. FRF estimators

\begin{tabular}{|c|c|l|}
\hline Estimator & Equation & \multicolumn{1}{c|}{ Properties } \\
\hline \hline$H_{1}(\omega)$ & $\frac{S_{X Y}(\omega)}{S_{Y Y}(\omega)}$ & $\begin{array}{l}\text { underestimation of amplitude, } \\
\text { good estimation in anti-resonance }\end{array}$ \\
\hline$H_{2}(\omega)$ & $\frac{S_{X X}(\omega)}{S_{Y X}(\omega)}$ & $\begin{array}{l}\text { overestimation of amplitude, } \\
\text { good estimation in resonance }\end{array}$ \\
\hline$H_{v}(\omega)$ & $\sqrt{H_{1}(\omega) H_{2}(\omega)}$ & $\begin{array}{l}\text { mix of } H_{1}(\omega) \text { and } H_{2}(\omega), \\
\text { minimize input and output noise }\end{array}$ \\
\hline
\end{tabular}

Resulting from the applicability of the RFP and LSCE method to linear systems, the coherence $\gamma$ of the signals has to be analyzed, too. If the signals are free from noise and error, and a linear system behavior being additionally existent; the $H_{1}(\omega)$ estimator is equal to the $H_{2}(\omega)$ estimator. In this case, the system causality has a maximum, and the coherence is equal to 1.

\subsection{Rational fractional polynomials method}

The Rational Fractional Polynomials (RFP) method is a frequency domain method. In this case, the model fit is performed in frequency domain. The basis for this method is the estimated FRF with the procedure described in Section 3.1. At the beginning, the analytical formulation of 
a FRF of a multi degree of freedom system is necessary. This transfer function can be expressed in Laplace domain using the complex argument $s=j \omega_{i}$

$$
H_{a}(s)=\sum_{k=1}^{n}\left(\frac{r_{k}}{s-p_{k}}+\frac{r_{k}^{*}}{s-p_{k}^{*}}\right)=\frac{\sum_{k=0}^{m} a_{k} s^{k}}{\sum_{k=0}^{n} b_{k} s^{k}}
$$

wherein, the number of pole pairs $p_{k}$, i.e. the number of degrees of freedom is described by $n$. In this context, $r_{k}, a_{i}$ and $b_{i}$ denote complex residues and coefficients of the analytical formulation of the FRF. In the next step, the error between the analytical formulation of the FRF and the measured data has to be calculated

$$
e_{i}=\sum_{k=0}^{m} a_{k}\left(j \omega_{i}\right)^{k}-h_{i}\left[\sum_{k=0}^{n-1} b_{k}\left(j \omega_{i}\right)^{k}+\left(j \omega_{i}\right)^{n}\right]
$$

Using $h_{i}$, the measured data of the FRF at the frequencies $\omega_{i}$ are defined. The square error criterion is specified as follows

$$
\sum_{i=1}^{L} e_{i}^{2} \rightarrow \min
$$

This error has to be minimized using the variables $a_{i}$ and $b_{i}$. Based on the calculated variables, the constant eigenfrequencies and the damping values can be determined.

\subsection{Least-squares complex exponential method}

The Least-Squares Complex Exponential (LSCE) method is a time domain method. Here, the fit is performed in the time domain. The basis within this method is the Inverse Fourier Transform (IRF) of the FRF formulated in frequency domain

$$
H_{a}(s)=\sum_{k=1}^{2 n} \frac{r_{k}}{s-p_{k}}
$$

In the next step, the IRF is calculated

$$
h_{a}(t)=\operatorname{ifft}\left(H_{a}(s)\right)=\sum_{k=1}^{2 n} r_{k} \mathrm{e}^{p_{k} t}
$$

This equation describes the structural response after impulse excitation. If the response is present at discrete time points, the exponential expression can be replaced by $z_{k}^{m}=\mathrm{e}^{p_{k} m \Delta t}$ using the $z$-transform. Based on this, it is possible to rewrite equation (3.5) as follows

$$
h_{a}(m \Delta t)=\sum_{k=1}^{2 n} r_{k} z_{k}^{m}
$$

Applying the Prony equation (He and Fu, 2001), the poles $p_{k}$ i.e. $z_{k}^{m}$ are estimated. This equation represents $z_{k}^{m}$ as roots of a polynomial with real coefficients

$$
c_{0}+c_{1} z+c_{2} z^{2}+\ldots+c_{2 n} z^{2 n}=0
$$

This equation is for each root equal to 0 . Using the several samples $m$ of the IRF data, it is possible to merge equation (3.6) with equation (3.7)

$$
\sum_{m=0}^{2 n} c_{m} h_{a}(m \Delta t)=0
$$


From this relationship, the coefficients $c_{m}$ can be determined directly. Using the calculated coefficients, the roots $z_{m}$ in equation (3.7) can be calculated. In the final step, the constant eigenfrequencies and the damping values can be determined by the poles of the system $z_{m}$.

It should be noted that in order to apply the RFP and the LSCE method, the input signal (excitation) and the output signal (measured decay) are necessary. In a next step, the FRF has to be estimated. From this, two noisy signals are used for parameter identification.

\section{Experimental tests}

In this Section, an experimental setup for damping evaluation is presented briefly, see Hentschel et al. (2014) for details. This setup delivers the necessary data to compare the methods for parameter estimation. It consists of a specimen with defined eigenfrequencies which is clamped in its nodes of vibration (regarding the analyzed mode shape). The structure is excited by a force impact using a voice coil actuator.

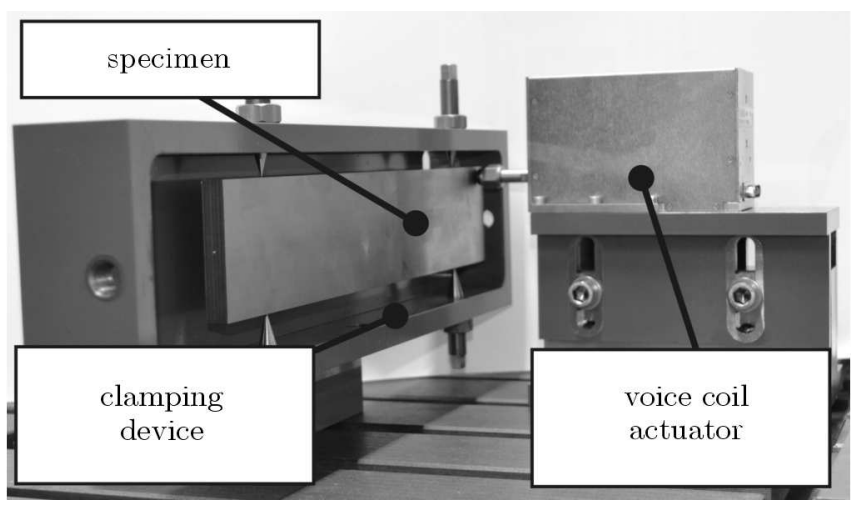

Fig. 10. Experimental setup

\subsection{Excitation and measurement}

The analyzed specimen is excited by a force impact generated by a voice-coil actuator. Hereby, an automated test sequence with a high reproducibility of the excitation force is realizable. In addition, no mass loading between excitation and structure is necessary. From this, a potential source for data distortion is eliminated. The voice-coil actuator is operating in current mode to control the acceleration of the actuator. The decaying velocity is measured by a laser vibrometer. The optical measurement technique is used to avoid the mechanical coupling between the sensor and the structure. In Fig. 11, the schematic experimental setup is presented. The excitation and the measurement are carried out at one point of the structure. In doing so, a system reduced to a single input and output is analyzed.

\subsection{Specimen clamping}

The investigated specimens are beams with defined eigenfrequencies. The sample geometry (width, length and thickness) is selected in a way so that the eigenfrequencies are well seperated. To reduce the influence of frictional damping due to clamping and to ensure a linear system behavior, the specimens are clamped in a suspended way, see Hentschel et al. (2014) for details. The beams are clamped in their nodes of vibration. Bolts with an apex are used to fix the specimens depending on the analyzed mode shape. This allows an evaluation of "free" modes of vibration and prevents rigid body mode shapes. 


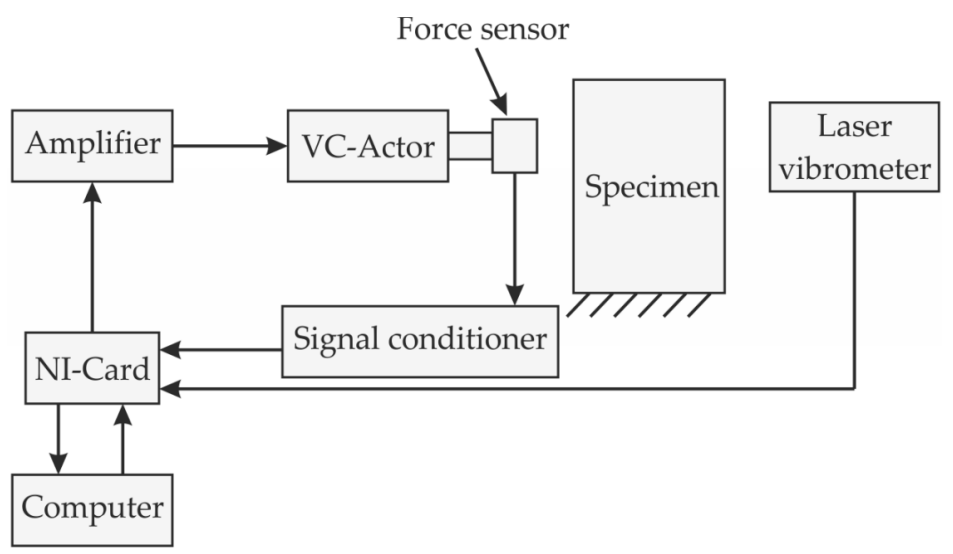

Fig. 11. Experimental setup (schematic)

\section{Evaluation}

To quantify the quality of the presented STFR method, the damping values identified using the different methods are compared. To apply the LSCE and the RFP method respectively, an estimation of the transfer function is necessary. As it is usual for modal analysis, the average of several transfer functions is performed, until a sufficient value of the identified coherence is present. For this purpose, an averaging of five measurements is performed. On this basis, the FRF is estimated using the $H_{2}(\omega)$ FRF estimator. Applying the LSCE and the RFP method, the damping values are calculated for the first two mode shapes of the specimen. To calculate a standard deviation of the identified damping values using the two common methods, the described procedure is repeated six times after reassembly of the experimental setup.

Using the performed thirty measurements to identify the damping by making use the RFP and the LSCE method respectively, the STFR method is applied to identify the damping values. Hereby, the decay curve is identified by means of the procedure described in Section 2.2. On the basis of the analytical formulation in equation (2.9), the least square method is applied to identify the decay coefficient. The mean damping values and the standard deviations calculated with the several methods are shown in Fig. 12.

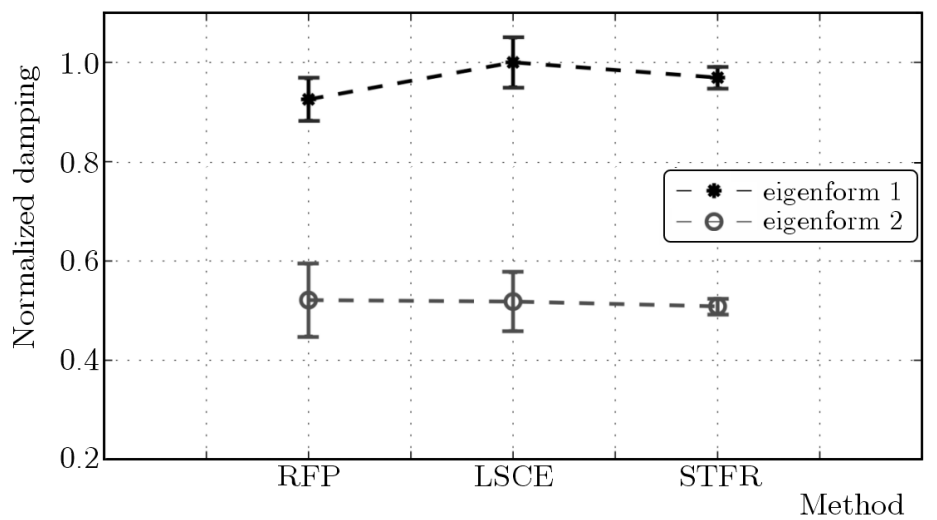

Fig. 12. Methods for damping determination

It can be seen that nearly the same mean damping values are calculated using the several methodologies. The standard deviations calculated on the basis of the RFP and the LSCE methods show almost equal magnitudes. However, the standard deviation found by using the STFR method is lower in comparison to the other methods. This difference arises from the calculation of the FRF which is necessary to apply the LSCE and the RFP method respectively. Besides the measured output signal, the measured input signal including noise and measurement errors are necessary, too. This is not the case applying the STFR method. 


\section{Conclusions}

In this paper, a method for the evaluation of damping values from experimental tests is presented. The presented method is limited to excited structures by an impulse with well separated eigenfrequencies. On this basis, resampling of the original data is introduced to achieve a sufficient quality concerning the calculated amplitude and frequency. An additional benefit of the applied resampling procedure is the identification of the present eigenfrequency depending of the vibration amplitude. To perform the damping identification, only the output signal is necessary in contrast to other common methods. For this purpose, determination of the transfer function is not necessary. In addition, the analysis of a given output signal is performed to clarify the approach for parameter identification. In this context, an amplitude-dependant damping and eigenfrequency is predefined. Hereby, the possibility of non-linear signal evaluation is demonstrated.

Finally, the presented methodology is compared to common methods used in linear modal analysis. Hereby, an analysis of experimentally identified damping values is performed. It can be shown that the intended method allows adequate parameter identification, in which only the output signal is used. Additionally, the identification of the amplitude dependent frequency and damping value is possible.

\section{References}

1. Bert C.W., 1973, Material damping: an introductory review of mathematical models measures and experimental techniques, Journal of Sound and Vibration, 29, 129-153

2. Feldman M., 1994, Non-linear system vibration analysis using Hilbert transform-II, forced vibration analysis method 'Forcevib', Journal of Mechanical Systems and Signal Processing, 8, 309-318

3. Hans S., Irbaim S., Pernot S., Boutin C., Lamarque C.H., 2000, Damping identification in multi-degree-of-freedom systems via a wavelet-logarithmic decrement - part 2: study of a civil engineering building, Journal of Sound and Vibration, 253, 375-403

4. He J., Fu Z.F., 2001, Modal Analysis, Butterworth Heinemann, Oxford, Auckland, Boston, Johannesburg, Melbourne, New Delhi

5. Hentschel O.P., Panning-von Scheidt L., Wallaschek J., Denk M., Masserey P.A., 2014, Influential parameters on structural damping values of turbine blades, Proceedings of ASME Turbo Expo 2014, Power for Land, Sea and Air, June 16-20, Düsseldorf, Germany, Paper GT201425656

6. Jinting W., Dandan L., Feng J., Chuhan Z., 2013, Accuracy of the half-power bandwidth method with a third-order correction for estimating damping in multi-DOF systems, Earthquake Engineering and Engineering Vibration, 12, 33-38

7. Lamarque C.H., Pernot S., Cuer A., 2000, Damping identification in multi-degree-of-freedom systems via a wavelet-logarithmic decrement - Part 1: Theory, Journal of Sound and Vibration, 253, 361-374

8. Lardies J., Gouttebroze S., 2002, Identification of modal parameters using the wavelet transform, International Journal of Mechanical Sciences, 44, 2263-2283

9. Le T.P., Argoul P., 2004, Continuous wavelet transform for modal identification using free decay response, Journal of Sound and Vibration, 277, 73-100

10. Meisner M., 2012, Accuracy issues of discrete Hilbert transform in identification of instantaneous parameters of vibration signals, Acoustic and Biomedical Engineering, 121, 164-167

11. Nelder J.A., Mead R., 1965, A simplex method for function minimization, The Computer Journal, 7, 308-313 
12. Oppenheim A.V., Schafer R.W., 2010, Discrete-Time Signal Processing, Third edition, Prentice Hall

13. Petrov E.P., Ewins D.J., 2006, Effects of damping and varying contact area at blade-disk joints in forced response analysis of bladed disk assemblies, Journal of Turbomachinery, 128, 403-410

14. Plunkett R., 1959, Measurement of Damping, Structural Damping, Ruzicka J., ed., ASME, Atlantic City, NJ USA, 117-131

15. Rao J.S., Saldanha A., 2003, Turbomachine blade damping, Journal of Sound and Vibration, 262, 731-738

16. Rice T., Bell D., Singh G., 2007, Identification of the stability margin between safe operation and the onset of blade flutter, Proceedings of ASME Turbo Expo 200\%, Power for Land, Sea and Air, May 14-17, Montreal, Canada, Paper GT2007-27462

17. Richardson M.H., Formeti D.L., 1982, Parameter estimation from frequency response measurements using rational fractional polynomials, Proceedings of the First International Modal Analysis Conference, 167-180, Orlando, USA

18. Siewert C., Panning L., Gerber C., Masserey P. A., 2008, Numerical and experimental damping prediction of a nonlinearly coupled low pressure steam turbine blading, Proceedings of ASME Turbo Expo 2008, Power for Land, Sea and Air, June 09-13, Berlin, Germany, Paper GT2010-51073

19. Slavič J., Simonovski I., Boltežar M., 2003, Damping identification using a continuous wavelet transform: application to real data, Journal of Sound and Vibration, 262, 291-307

20. Yang Y., Cascante G., Polak M. A., 2011, New method for the evaluation of material damping using the wavelet transform, Journal of Geotechnical and Geoenvironmental Engineering, 137, 798-808

21. Yang J.N., Lei Y., Pan S., Huang N., 2003a, System identification of linear structures based on Hilbert-Huang spectral analysis. Part 1: Normal modes, Earthquake Engineering and Structural Dynamics, 32, 1443-1467

22. YAng J.N., Lei Y., PAn S., Huang N., 2003b, System identification of linear structures based on Hilbert-Huang spectral analysis. Part 2: Complex modes, Earthquake Engineering and Structural Dynamics, 32, 1533-1554 